\title{
Laser optoacoustic imaging of turbid media: determination of optical properties by comparison with diffusion theory and Monte Carlo simulation
}

Alexander A. Oraevsky, Rinat O. Esenaliev, Frank K. Tittel, Martin R. Ostermeyer, Lihong V. Wang, et al.

Alexander A. Oraevsky, Rinat O. Esenaliev, Frank K. Tittel, Martin R. Ostermeyer, Lihong V. Wang, Steven L. Jacques, "Laser optoacoustic imaging of turbid media: determination of optical properties by comparison with diffusion theory and Monte Carlo simulation," Proc. SPIE 2681, Laser-Tissue Interaction VII, (7 May 1996); doi: 10.1117/12.239584

SPIE. Event: Photonics West '96, 1996, San Jose, CA, United States 
Laser opto-acoustic imaging of turbid media: determination of optical properties

by comparison with diffusion theory and Monte Carlo simulation

\author{
Alexander Oraevsky ${ }^{1}$, Rinat Esenaliev ${ }^{1}$, Frank K. Tittel ${ }^{1}$ \\ Martin Ostermeyer ${ }^{2}$, Lihong Wang ${ }^{2}$, Steven L. Jacques ${ }^{2}$,
}

1. Department of Electrical and Computer Engineering, Rice University, Houston, TX 77251

2. Laser Biology Research Laboratory, UT / MD Anderson Cancer Center, Houston, TX 77030

\begin{abstract}
Laser optoacoustic imaging based on time-resolved detection of laser-induced thermoelastic pressure waves can be potentially used in medicine as a diagnostic tool (tomography) and as means to measure tissue optical properties in vivo. Information on tissue optical properties in vivo is important for laser dosimetry and tissue characterization. Analysis of the profile and amplitude of laser-induced transient stress can provide direct information about absorbed energy distribution in irradiated volume. Pressure wave profiles exactly correspond to temperature distributions generated in tissues under irradiation conditions of temporal stress confinement. In this study we experimentally measured pressure wave profile and amplitude upon irradiation of highly scattering gel phantoms and aqueous solutions colored with potassium chromate and made turbid with polystyrene spheres. A wide-band lithium niobate acoustic transducer was used to detect laser-induced pressure waves. The experimentally measured laser-induced stress profiles and the results of a Monte Carlo simulation were in excellent agreement. Computer code was developed to calculate separately absorption and scattering coefficients from transient stress profiles. Our study has demonstrated feasibility of time-resolved detection of laser-induced acoustic transients as a method for tissue optical properties measurements in vivo.
\end{abstract}

Key Words: Acoustic transducer, laser-induced stress, optoacoustic imaging, tissue optical properties, light distribution.

\title{
1. LASER-INDUCED STRESS AS A METHOD TO LOOK THROUGH TURBID MEDIA
}

The generation of acoustic waves by the consecutive transformation of light energy into heat and then to mechanical stress is referred to as the thermo-optical mechanism of stress generation [1]. Short laser pulses allow the most efficient generation of thermo-optical stress. The absorption of laser radiation in a medium is usually followed by a fast heat generation. Subsequently, thermal expansion of the instantaneously heated tissue causes a pressure rise, $\Delta \mathrm{P}$, in the irradiated volume. Due to the fact that thermal energy is deposited instantly, thermoelastic stress profile mirrors exactly steady-state light distribution in irradiated medium. After generation, transient stress wave with profile that resemble profile of light distribution propagate with the speed of sound in medium and can be detected by acoustic transducer. Profile of a planar acoustic wave propagating in medium along laser beam axis, $\mathrm{z}$, is the most accurate source of information about medium optical properties. In case when speed of sound in medium is known and piezoelectric detector has sufficient temporal resolution, electric signal, $S(t)$, displayed on the screen of digital scope can be converted into z-axial profile of absorbed laser energy:

$$
S(t)[\mathrm{mV}]=\Delta P(z)[\mathrm{bar}] S_{o}[\mathrm{mV} / \mathrm{bar}]=5 \Gamma(\mathrm{T}) \mu_{\mathrm{a}} \mathrm{H}(\mathrm{z}) \mathrm{T} \mathrm{R} D \Lambda S_{\mathrm{o}}\left(\mathrm{z}=\mathrm{c}_{\mathrm{s}} \mathrm{t}\right)
$$

where $\Gamma$ is the dimensionless Grüneisen parameter proportional to the fraction of thermal energy converted into mechanical stress, $\mathrm{H}\left[\mathrm{J} / \mathrm{cm}^{2}\right]$ is the laser irradiance distribution within tissue, $\mu_{\mathrm{a}}\left[\mathrm{cm}^{-1}\right]$, is the absorption coefficient of the medium. Pressure measured in $\left[\mathrm{J} / \mathrm{cm}^{3}\right]$ can be equally expressed in decabar. 
Dimensionless parameters $T, R, D$, and $\Lambda$ represent correction factors that account for transmission of acoustic wave through interfaces, stress relaxation during laser energy deposition, diffraction, and acoustic attenuation respectively, and $S_{0}$ is the sensitivity of acoustic transducer.

If a wide diameter laser beam with a top-hat radial energy distribution is used to induce a planar acoustic wave in the irradiated medium, only the longitudinal (z-axial) distribution of thermoelastic stress may be considered. The temporal profile of the acoustic signal corresponds to the initial $\mathrm{z}$-axial distribution of the absorbed laser energy and can be monitored by an acoustic transducer with sufficient temporal resolution. The rise-time of both the transducer and the digital oscilloscope must be shorter than the duration of the stress transients, $1 / \delta c_{s}$.

The amplitude of the time-resolved acoustic signal is proportional to the medium absorption coefficient, $\mu_{\mathrm{a}}$. Profile of laser irradiance $\mathrm{H}(\mathrm{z})$ can not be exactly described by analytical expression due to the influence of diffused back scattering on the profile of light distribution in turbid medium. However, $\mathrm{H}(\mathrm{z})$ can be presented as a simple exponent $\exp (-\mathrm{z} / \delta)$ at the depth in medium equal to about two meanfree-paths of light. Detailed treatment of equation (1) is given in [2].

Therefore, both optical properties of turbid medium can be determined from laser-induced stress profile. The purpose of this paper is twofold: to compare time-resolved profiles of laser-induced stress with Monte Carlo simulation of light distribution in absorbing turbid medium with known optical properties, and to employ diffusion theory for determination of medium optical properties.

\section{MATERIALS and METHODS}

A non-scattering, homogeneously absorbing aqueous solution of potassium chromate $\left(\mathrm{K}_{2} \mathrm{CrO}_{4}\right)$ was used to as an absorbing liquid. Absorption coefficient of aqueous potassium chromate was measured with spectrophotometer and verified with time-resolved detection of laser-induced stress. Measurement of absorption coefficient in colored water is a straight forward procedure which yields very accurate information in case of planar acoustic wave generation by a wide beam of a short laser pulse. It was not difficult to make all the correction parameters involved in equation (1) to equal 1 in our reference solution. However, exponential slope of laser-induced stress profile in this simple case will not be altered even in case when any or all correction parameters are $<1$. Exponential fit of transient stress yields very accurate number for absorption coefficient. To understand how accurate is this measurement, one can determine absorption coefficient from large number of spectrophotometer measurements in one and the same solution with different optical thickness. Set of spectrophotometer data in solutions with various thickness is similar to the laser-induced stress profile measured with acoustic transducer with sufficient temporal resolution. Absorption coefficient measured in reference aqueous solution was $5.78 \mathrm{~cm}^{-1}$. A $2 \%$ polystyrene sphere solution have a scattering coefficient, $\mu_{s}$, of $1218 \mathrm{~cm}^{-1}$ and an anisotropy, $\mathrm{g}$, of 0.918 at $355 \mathrm{~nm}$, yielding a reduced scattering coefficient, $\mu_{s^{\prime}}$, of $111.8 \mathrm{~cm}^{-1}$ at $355 \mathrm{~nm}$. Final experimental solution with pre-measured optical properties was made by mixing equal volumes of potassium chromate and polystyrene spheres aqueous solutions. For future references let us call this solution as PCPSA solution. Optical properties of the final PCPSA solution were as follows: $\mu_{\mathrm{a}}=2.89 \mathrm{~cm}^{-1}, \mu_{\mathrm{s}}{ }^{\prime}=55.9 \mathrm{~cm}^{-}$ 1 . The ratio of $\mu_{\mathrm{s}} / \mu_{\mathrm{a}}$ was chosen in the range typical for biological tissues.

The samples were irradiated by a Nd:YAG laser operated in a Q-switched mode (YG 681, Continuum, Santa Clara, CA). Experiments were conducted at the wavelength of $355 \mathrm{~nm}$ to match the absorption band of potassium chromate. The energy of each laser pulse was measured with a calibrated joulemeter (ED-200, Gentec, Canada). A $5 \mathrm{~mm}$ IRIS aperture was used to select the most homogeneous part of the laser beam for tissue irradiation and produce top-hat radial energy distribution. The temporal 
pulse-shape was nearly Gaussian with a full width of $11.8 \mathrm{~ns}$ at $1 / \mathrm{e}$ of its amplitude. A wide-band acoustic transducer (WAT-13, Science Brothers Inc., Houston, Texas) with nanosecond resolution was used to measure absolute amplitudes and profiles of transient stress waves in transmission mode [3].

\section{EXPERIMENT}

Fig. 1 displays laser-induced stress profile in PCPSA solution covered with a quartz slab. Quartz produced rigid boundary that helped to clearly determine position of solution surface as a minimum in temporal stress profile. We have set time zero at the moment when stress generated at the surface reached acoustic transducer located at the bottom of cuvette with PCPSA solution. Therefore, the profile of stress wave generated within aqueous solution was recorded at moments on the negative time-scale.

\section{Solution with polystyrene spheres colored with $\mathrm{K}_{2} \mathrm{CrO}_{4}$}

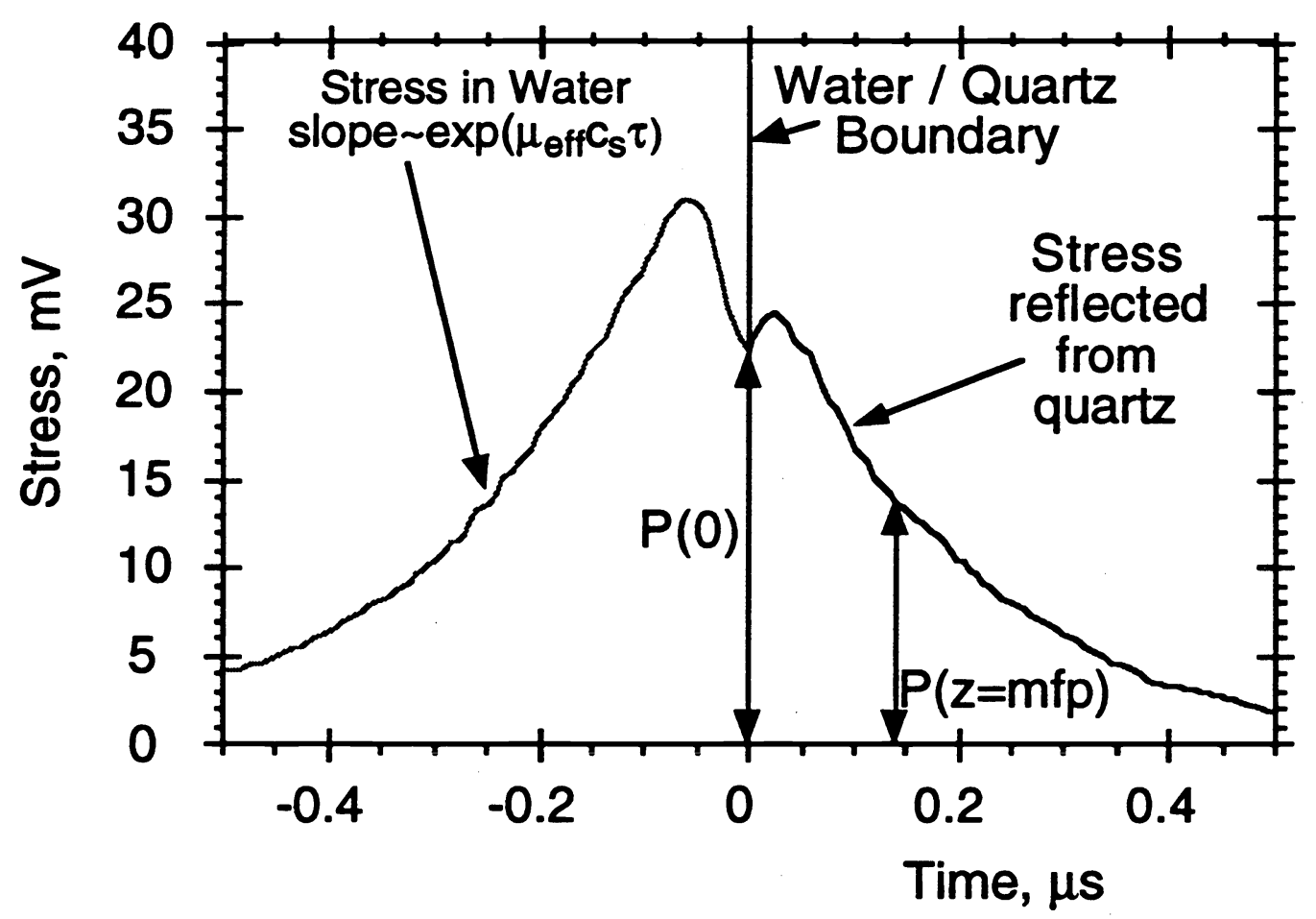

EIGURE 1. Typical stress profile measured in a layer of aqueous solution with $2 \mathrm{~mm}$ thickness. Quartz slab with $5 \mathrm{~mm}$ thickness was used to produce rigid acoustic boundary.

Fig. 1 shows that the profile of absorbed laser energy in solution with dominant scattering has a maximum in subsurface layer. This profile can not be described analytically. Complex shape of subsurface energy distribution presents the major problem for direct measurements of optical properties in turbid tissues with TRSD method. Absorption coefficient in a turbid medium can be measured from the acoustic transient profile only if the subsurface irradiance is known. The are two ways to overcome the problem of uncertainty of subsurface fluence value. One possibility is to employ iterative computer simulations (based on Monte Carlo) to deduce optical properties directly from measured stress profile. The other possibility is to use the total diffuse reflectance measurement to complement the TRSD method 
for the determination of either the subsurface fluence in turbid media or the ratio of absorption and scattering coefficients [6].

Nevertheless, diffusion theory can describe accurately profile of absorbed energy distribution in homogeneous turbid medium at the depth starting with one-two transport mean free paths. Therefore, our approach was to employ diffusion theory to deduce both optical properties of PCPSA solution from timeresolved profile of laser-induced stress.

\section{Comparison of Laser-Induced Stress Profile with Monte Carlo}

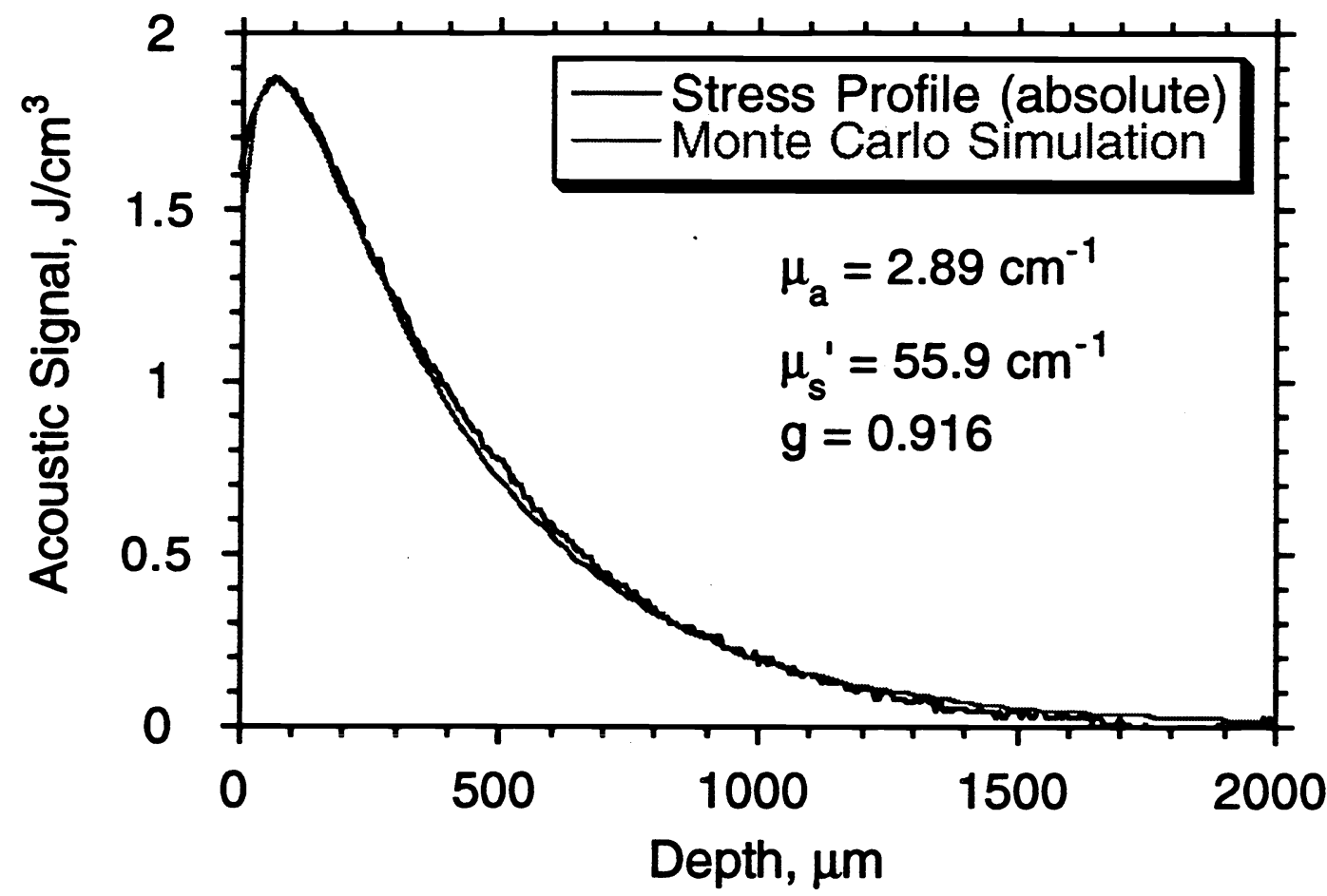

EIGURE 2. Laser-induced stress profile that resemble absorbed laser energy distribution in "reference" aqueous solution of potassium chromate with polystyrene spheres. Monte Carlo simulation of absorbed light distribution is also presented based on pre-measured optical properties of "reference" PCPSA solution.

First of all, we compared the profile of laser-induced stress with Monte Carlo simulation. Axial Monte Carlo simulation calculates distribution of photons along $\mathrm{z}$-axis of laser beam propagation in medium yielding numbers in $[\mathrm{J} / \mathrm{cm}]$. Absolute measurements of stress yield z-axial profiles in $\left[\mathrm{J} / \mathrm{cm}^{3}\right]$. Therefore, we scaled axial Monte Carlo profile to match amplitude of measured stress in subsurface layer. Two superimposed profiles are shown in Fig. 2. Results of comparison were impressive yielding deviations of one profile from the other within $5 \%$ margin.

\section{MODEL}

We have used a diffusion theory model to reconstruct the optical properties from the measured absorbed energy profile. The geometry of the diffusion theory model for a pencil beam incident on a semi infinite medium is depicted in Fig. 3 . Using the extrapolated boundary condition $[4,5]$, a pencil beam can 
be modeled by a buried point source at a depth $z_{\text {source }}=3 D$, where $D=1 / 3 /\left(\mu_{\mathrm{a}}+\mu_{\sigma}{ }^{\prime}\right)$ is the diffusion coefficient. The source strength of this equivalent point source is the power of the incident beam $\mathrm{P}_{0}$ scaled by the transport albedo $\mathrm{a}^{\prime}=\mu_{\sigma}{ }^{\prime} /\left(\mu_{\mathrm{a}}+\mu_{\sigma}{ }^{\prime}\right)$. To satisfy the extrapolated boundary condition a negative image source is added at $z_{\text {image }}=-4 A D-3 D$.

The fluence $H$ at a point on the $\mathrm{z}$-axis due to a laser beam that enters the tissue at a radial distance $\rho$ from the $\mathrm{z}$-axis can be expressed as:

where

$$
H(z, r)=P_{0} \frac{a^{\prime}}{4 p D}\left(\frac{e^{-r_{1} / d}}{r_{1}}-\frac{e^{-r_{2} / d}}{r_{2}}\right) ; \quad(z \geq 3 D)
$$

$$
r_{1}=\sqrt{(z-3 D)^{2}+\rho^{2}} \quad ; \quad r_{2}=\sqrt{(z+3 D+4 A D)^{2}+\rho^{2}} ; \delta=\sqrt{D / \mu_{a}} .
$$

The constant $A=\left(1+R_{\text {eff }}\right) /\left(1-R_{\text {eff }}\right)$ accounts for the effective reflection $R_{\text {eff }}$ due to a mismatch of the refractive indices of the tissue and the outer medium [4].

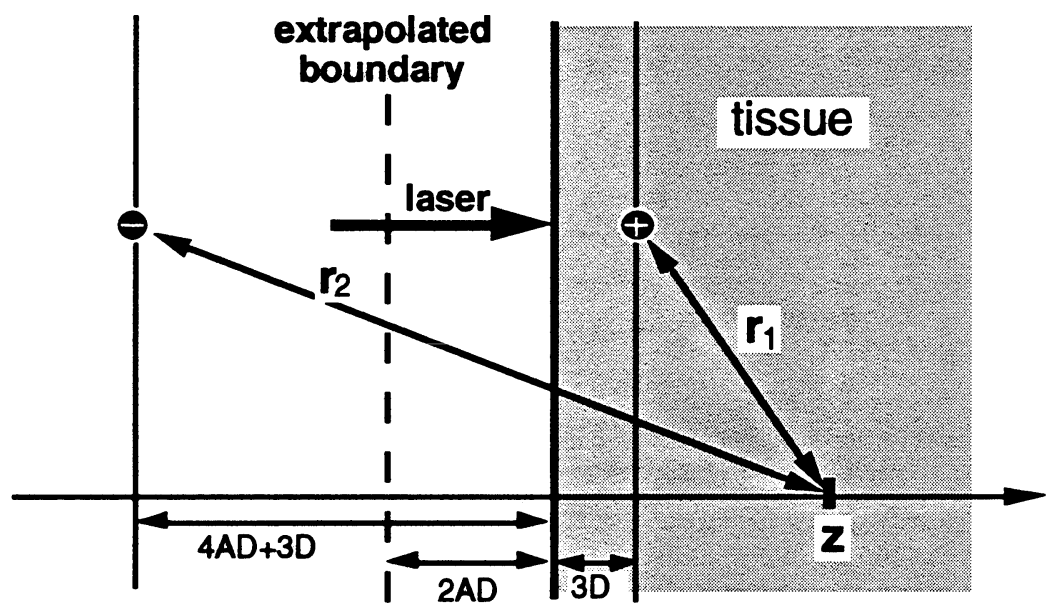

FIGURE 3. Geometry of the diffusion theory model of for a pencil beam incident on a semi infinite medium.

To model a broad beam illumination we integrate equation (2) over the beam area. The analysis is simplified by assuming an infinitely broad beam (which was quite accurate approximation for our experimental conditions).

$$
H(z)=2 \pi \int_{0}^{\infty} H(z, \rho) \rho d \rho=H_{0} a^{\prime} \frac{\delta}{2 D}\left(e^{3 D / \delta}-e^{-(3 D+4 A D) / \delta}\right) \cdot e^{-z / \delta}
$$

This yields the absorbed energy $\mathrm{Q}(\mathrm{z})=\mu_{\mathrm{a}} \mathrm{H}(\mathrm{z})$ as a function of depth

$$
Q(z)=H_{0} k(D, \delta, A) e^{-z / \delta}
$$


with

$$
k(D, \delta, A)=\left(\frac{1}{2 \delta}-\frac{3 D^{2}}{2 \delta^{3}}\right) \cdot\left(e^{3 D / \delta}-e^{-(3 D+4 A D) / \delta}\right),
$$

where we have expressed $\mu_{\mathrm{a}}$ and $\mathrm{a}$ ' in terms of the diffusion coefficient $\mathrm{D}$ and the penetration depth $\delta$.

To reconstruct the optical properties from the acousto-optical measurement of $Q(z)$ we use the following approach: A scaled exponential function with offset is fitted to the normalized measurement data.

$$
\frac{Q(z)}{H_{0}}=k_{f i t} e^{-z / \delta_{s t}}+c
$$

The constant $c$ accounts for experimental offset errors and is not used further. To determine the diffusion coefficient $D$ from the fitted scaling factor $k_{\text {fit }}$ we solve the implicit equation:

$$
0=k\left(D, \delta_{f i t}, A\right)-k_{f i t}
$$

numerically for $\mathrm{D}$. The absorption and reduced scattering coefficients can then be computed from $\mathrm{D}$ and $\delta_{\text {fit }}$ Equation (7) has generally two roots, however, the larger value can be discarded, since it does not fulfill the restriction $\mu_{a} \ll<\mu_{\sigma}^{\prime}$ for the validity of the diffusion approximation.

It is important to note that near the surface equation (4) is not applicable because the diffusion approximation fails near sources. Therefore only data for depths $\mathbf{z}_{\min }$ larger than about two transport mean free paths (equal to 6D) should be used for the fit. However, the optical properties are not known in advance. A practical approach is to plot the measurement data in logarithmic scale, and fit the data from a depth were the curve "looks straight". If the reconstructed optical properties indicate that the $\mathrm{z}_{\min }>6 \mathrm{D}$ condition is violated, the procedure is repeated with a larger $\mathrm{z}_{\min }$, until the condition is met.

\section{RESULTS}

A liquid phantom (PCPSA solution) with optical properties $\mu_{\mathrm{a}}=2.89 \mathrm{~cm}^{-1}$ and $\mu_{\sigma}{ }^{\prime}=55.96 \mathrm{~cm}^{-1}$ was prepared. Groenhuis' formula [4] was used to compute the boundary factor A $=2.9$ from the refractive index of water $n(355 \mathrm{~nm})=1.343$.

Experimental data were measured with the acoustic transducer and normalized (see Fig 4). The reconstruction algorithm determined the optical properties as $\mu_{\mathrm{a}}=2.26 \mathrm{~cm}^{-1}$ and $\mu_{\sigma}^{\prime}=53.4 \mathrm{~cm}^{-1}$. The relative error compared to the known optical properties is $-22 \%$ and $-4 \%$ for absorption and reduced scattering respectively.

Additionally, a data set was created with a Monte Carlo simulation using the known optical properties of the phantom. The reconstruction algorithm yielded $\mu_{\mathrm{a}}=2.894 \mathrm{~cm}^{-1}$ and $\mu_{\sigma}{ }^{\prime}=54.29$. The relative error compared to the known optical properties is $0.1 \%$ and $-2.9 \%$ for absorption and reduced scattering respectively.

\section{DISCUSSION}

The small error in the reconstruction of optical properties from the simulated Monte Carlo data shows that the method is principally sound. We attribute the relatively large error in the reconstruction from the 
measured data to the absolute calibration error for the acoustic transducer. Also Grüneisen coefficient for PCPSA solution can differ from that of water. The penetration depth can be determined with high accuracy from the shape of the measured profile. The diffusion coefficient, $D$, can be determined from the scaling factor of the fitted exponential function. Therefore the calibration accuracy of the acoustic transducer and accurate determination of all correction factors for time-resolved measurement of stress profiles are directly related to the accuracy of the deduced value for $D$.

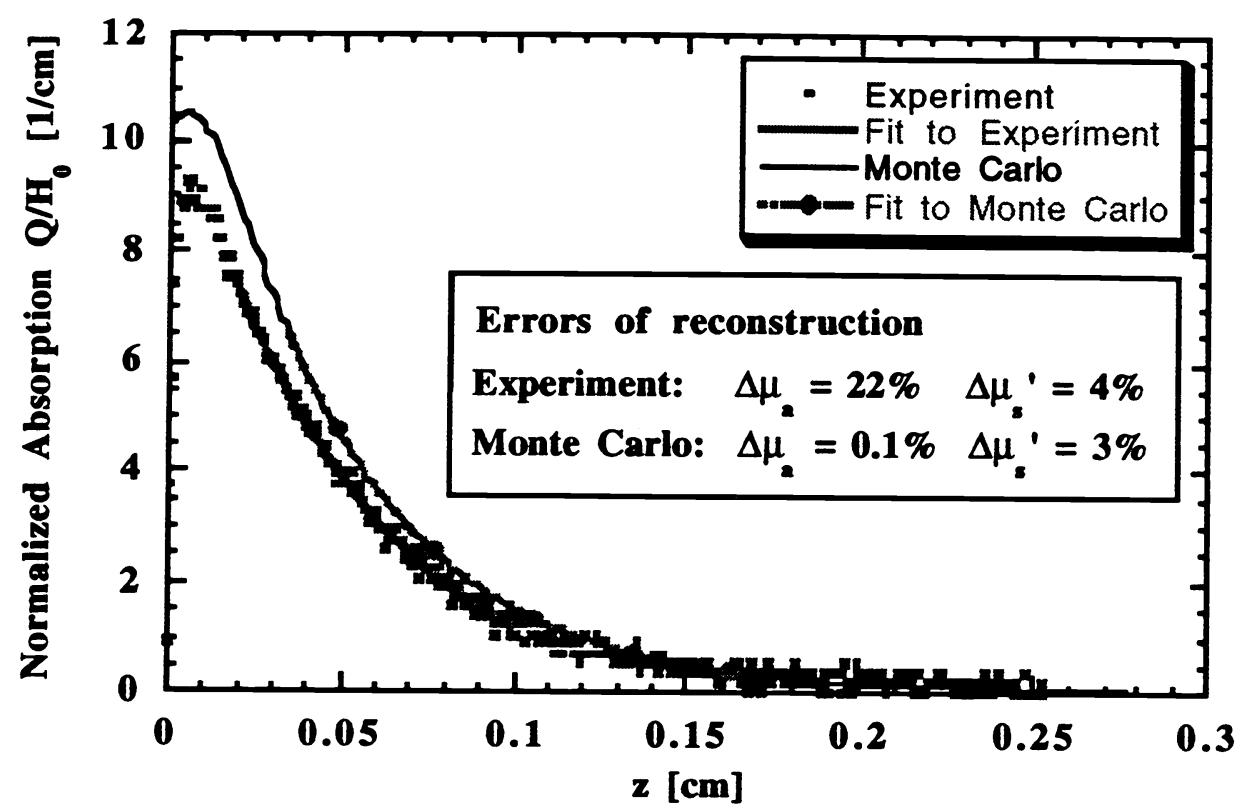

EIGURE 4. Reconstruction of optical properties from measured data and Monte Carlo simulated data for a phantom with absorption coefficient $\mu_{\mathrm{a}}=2.89 \mathrm{~cm}^{-1}$ and reduced scattering coefficient $\mu_{\sigma}{ }^{\prime}=55.96 \mathrm{~cm}^{-1}$.

\section{CONCLUSION}

An acoustic signal induced by a laser pulse under confined stress conditions displays z-axial temperature distributions which may be used for imaging and measurement of tissue optical properties. The TRSD method requires much shorter data acquisition time and has a much higher dynamic range as compared with the photon detection techniques. The main advantage of the time-resolved laser optoacoustic technique is the capability to determine both optical coefficients from one measurement of laserinduced acoustic signal at one location on the tissue surface in vivo. Unlike other methods that measure averaged optical properties of biological tissues over the irradiated volume, the time-resolved detection of laser-induced stress can display the exact $\mathbf{z}$-axial profile of the absorbed energy distribution in layered media [7] and media with distributed heterogeneities [8].

On the other hand, deduction of optical properties from TRSD measurements in the ranges of acoustic frequencies where diffraction and attenuation may not be neglected can be more complicated. The diffraction of acoustic waves is prominent for low acoustic frequencies (low light absorption coefficients) and narrow laser beams (beam diameter is less than the light penetration depth). For high acoustic frequencies diffraction is not significant, although attenuation occurs. Most media including biological tissues have insignificant acoustic attenuation in the low frequency range where diffraction plays a considerable role. In contrast, attenuation is significant for high acoustic frequencies where diffraction may be neglected. This allows us to study separately these two effects that alter amplitude and profile of 
the detected acoustic signal. In addition, future work will include measurements of Grüneisen coefficients that determine fraction of laser energy converted into stress for biological tissues.

\section{ACKNOWLEDGMENTS}

This research was supported by the U.S. Department of Energy (DE-FG05-91ER617226) and the Whitaker Foundation.

\section{Z. REEERENCES}

1. Gusev VE, Karabutov AA: "Laser Optoacoustics", AIP Press, New York, 1992.

2. Oraevsky AA, Jacques SL, Tittel FK: Determination of tissue optical properties by piezoelectric detection of laser-induced stress waves, Proc. SPIE 1993; 1882: 86-101.

3. Oraevsky AA: A nanosecond acoustic transducer with applications in laser medicine, IEEE/LEOS Newsletter 1994; 8(1): 6-17.

4. Farrell TJ, Patterson MS, Wilson B: A diffusion theory model of spatially resolved steady state diffuse reflectance for the noninvasive determination of tissue optical properties in vivo, Med. Phys. 1992; 19: 879-888.

5. RC Haskell RC, Svaasand LO, Tsay TT, Feng TC, McAdams MS, Thromberg BJ: Boundary conditions for the diffusion equation in radiative transfer, J. Opt. Soc. Am A 1994; 11: 2727-2741.

6. Oraevsky AA, Jacques SL, Tittel FK: Measurement of tissue optical properties by time-resolved detection of laser-induced transient stress, JOSA B, 1996; special issue on biomedical optics.

7. Oraevsky AA, Jacques SL, Esenaliev RO, Tittel FK: Radial and z-axial resolution in laser optoacoustic imaging with ultrasonic transducers, Proc. SPIE 1995; 2389: 198-208.

8. Karabutov AA, Letokhov VS, Podymova NV: Time-resolved opto-acoustic tomography of inhomogeneous media, Proc. SPIE 1995; 2389: 209-217. 